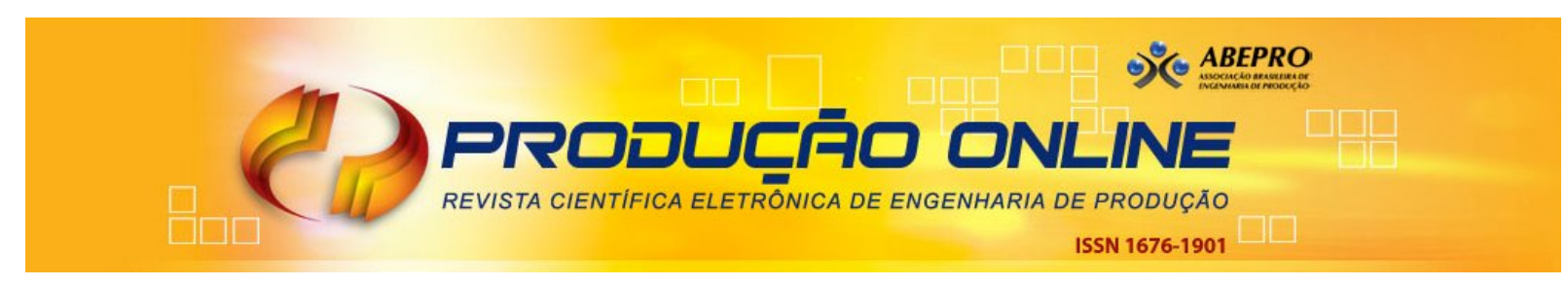

\title{
APLICAÇÃO DA TEORIA DAS FILAS EM SERVIÇOS BANCÁRIOS
}

\section{APPLICATION OF QUEUING THEORY IN BANKING SERVICES}

\author{
Vitor Costa de Lima* E-mail: vitor.costa08@gmail.com \\ Rosley Anholon* E-mail: rosley@fem.unicamp.br \\ Izabela Simon Rampasso* E-mail: izarampasso@gmail.com \\ Osvaldo Luiz Gonçalves Quelhas*^ E-mail: quelhas@latec.uff.br \\ *Universidade Estadual de Campinas (UNICAMP), Campinas, SP, Brasil \\ **Universidade Federal Fluminense (UFF) Niterói, RJ, Brasil
}

\begin{abstract}
Resumo: Tendo em vista a competição acirrada presente na economia nos dias atuais e a necessidade crescente de conquistar clientes e mantê-los fiéis, o presente trabalho tem como objetivo aplicar a consagrada Teoria das Filas em uma agência bancária com a finalidade de apontar oportunidades de melhorias no tempo de espera dos clientes, a partir do pressuposto de que as filas analisadas seguem a distribuição de Poisson. Tal pressuposto é decorrente da comparação realizada entre a referida distribuição com os dados de chegada dos clientes em todas as filas analisadas. Constatou-se também a existência de filas com sazonalidade na demanda, enquanto outras apresentaram uma demanda regular. A partir do estudo realizado, melhorias no gerenciamento desta agência bancária poderão ser realizadas como, por exemplo, a realocação de funcionários em determinados períodos para funções com maior demanda. Concluiu-se, portanto, que a aplicação da teoria é viável e que pode auxiliar no aumento das vantagens competitivas da agência.
\end{abstract}

Palavras-chave: Teoria das Filas. Análise de Serviços. Setor bancário.

Abstract: Bearing in mind the intense competition in the current economy and the increasing necessity of having more customers and keeping them loyal, this paper aims to apply the well known Queuing Theory in a bank agency to identify improvement opportunities in customer waiting time, from the assumption that the analyzed lines follow the Poisson's distribution. This assumption results from the comparison between such distribution to the customers' arrival data in all analyzed lines. It was also found that there are queues with seasonality in demand, while others showed a regular demand. From the study performed, improvements in the management of this bank agency may be made, for example, the reallocation of staff in certain periods for functions with higher demand. It was concluded, therefore, that the application of the theory is feasible and can help to increase the competitive advantages of the agency.

Keywords: Queuing Theory. Analysis Service. Banking sector.

\section{INTRODUÇÃO}

No atual mundo globalizado, "cabem aos bancos três importantes papéis na sociedade: 1) proteger e rentabilizar os investimentos dos indivíduos e das empresas; 2) financiar o consumo e o investimento; 3) prover serviços de pagamento e de recebimento" (BARBOSA, 2010). 
De acordo com o Banco Central (2015), os bancos comerciais têm como "objetivo principal proporcionar suprimento de recursos necessários para financiar, em curto e em médio prazo, o comércio, a indústria, as empresas prestadoras de serviços, as pessoas físicas e terceiros em geral".

Em meio à importância dos bancos, a busca pela eficiência dos processos neste segmento tornou-se importante (FREITAS E MORAIS, 2012). Para tanto, fazse necessária uma análise entre os principais agentes do segmento: as agências bancárias e seus clientes. Partindo de uma abordagem mais ampla, segundo Negretto (2007, p. 7), a "competitividade do mercado e a acessibilidade à informação têm provocado também mudanças no comportamento dos consumidores que se tornam cada vez mais exigentes e menos fiéis às empresas".

Diante deste cenário, "os bancos precisam melhorar sua capacidade de manter clientes e descobrir novas maneiras de tornar a carteira mais lucrativa ao longo do período de relacionamento com cada um deles" (OLIVEIRA, 2008, p. 32). Conforme Negretto (2007) e Oliveira (2008), as organizações se depararam com uma situação instável diante de um mercado exigente e mutável, portanto, o relacionamento com clientes e a retenção dos mesmos se tornaram fundamentais.

Atualmente os bancos procuram descobrir novos clientes, novos meios de conceder crédito ou de estabelecer vínculos com clientes e outros bancos. Procuram obter recursos em condições mais favoráveis, ampliando seu lucro, e, para tanto, o aprimoramento do serviço é essencial (FREITAS E MORAIS, 2012). O fato de ser muito concentrado, devido às economias de escala e de escopo existentes, acentua ainda mais a questão da competitividade do setor bancário (PAULA et al., 2013).

A criação diária de novos produtos é uma manifestação dessa busca por competitividade, que tem como objetivo principal garantir a satisfação dos clientes, o que acarreta em fidelização dos mesmos e assegura a lucratividade da empresa (JUNIOR e BORNIA, 2011). Segundo o SEBRAE (2012), a competitividade pressupõe produtos e/ou serviços de qualidade, além de bons preços.

A Teoria das Filas está diretamente relacionada com a realidade de uma agência bancária, tendo em vista as freqüentes filas neste ambiente. As filas que os clientes enfrentam para utilizar um serviço bancário refletem a falta de foco no cliente (ARAUJO et al., 2003; SHEIKH et al., 2013; XIAO e ZHANG, 2010). Sabbadini et al. (2006), Mulato e Oliveira (2006), Araújo et al. (2004), Ferreira et al. 
(2007), Fernandes e Marins (2012) e Pergher et al. (2014) destacam a necessidade de gerenciamento das filas.

Segundo Dávalos (2012, p. 4) a teoria parte do seguinte pressuposto "a formação de filas excede a capacidade de fornecer determinado serviço" e envolve o estudo matemático utilizando ferramentas de tratamento estatístico ou estocástico. Verifica-se que o estudo de serviços bancários, por meio da Teoria das Filas, pode proporcionar uma ferramenta poderosa para gestão dos processos em busca da prestação de serviços de excelência gerando satisfação, fidelidade e, consequentemente, mais negócios e menos custos para o banco.

Mediante ao exposto, este artigo pretende responder ao seguinte problema de pesquisa: como a aplicação da Teoria das Filas pode melhorar os processos cotidianos de uma agência bancária? A hipótese que se faz inicialmente, baseada na literatura da área, é de que a aplicação dessa teoria pode proporcionar vantagens competitivas à agência analisada, mas isso será comprovado ou refutado ao longo da pesquisa.

Para responder a tal questão, derivam-se três objetivos específicos:

a) mapear as atividades cotidianas de uma agência bancária e fazer um levantamento dos dados de ocorrência;

b) aplicar a Teoria das Filas;

c) analisar criticamente os resultados obtidos.

Destaca-se o fato de que o presente trabalho não questiona a aplicabilidade ou não da Teoria de Filas no setor bancário, pois se valendo dos pressupostos apresentados por Xiao e Zhang (2010); Ullah, Iqbal, Zhang e Ayat (2014); Yakubu e Najim (2014); Chowdhury (2013); Bandeira e Rocha (2010), os autores deste artigo entendem que sua utilização neste tipo de aplicação já é conhecida. A partir do pressuposto de que as filas estudadas seguem a distribuição de Poisson, é feita uma análise crítica dos dados levantados e proposição de melhorias.

\section{REVISÃO BIBLIOGÁFICA}

\subsection{Definição Teoria das Filas e exemplos de aplicação correlatos}

Filas são experiências indesejáveis (GROSS et al., 2008; CARDOSO et al., Revista Produção Online, Florianópolis, SC, v. 16, n. 1, p. 210-241, jan./mar. 2016. 
2010; ROMERO et al., 2010). Seja no trânsito de veículos, no supermercado, em ligações telefônicas ou, como no caso em questão, em agências bancárias. $E$ isto se deve ao fato da demanda por serviço ser maior que a capacidade de prestação do mesmo (GROSS et al., 2008).

A Teoria das Filas é uma modelagem analítica que aborda as filas por meio de fórmulas matemáticas (SHEIKH et al., 2013; PRADO, 2014), caracterizando as filas pelo tempo de espera e tempo de atendimento, aliado a características peculiares como capacidade de atendimento, capacidade máxima do sistema, tamanho da população e disciplina da fila (PRADO, 2014).

Como exemplo de uma aplicação bem sucedida da Teoria das Filas pode-se mencionar o trabalho de Figueiredo e Rocha (2010), que aplicaram a mesma em uma loja de departamento de grande porte. Neste artigo, os referidos autores coletaram os dados (tempo médio entre chegadas e tempo de atendimento) para visualização da característica da fila única e em seguida utilizaram-se da referida teoria para reconhecer padrões de demanda, permitindo a provisão e tomada de decisões com maior eficiência.

Um segundo exemplo de aplicação da Teoria das Filas é o artigo de Cardoso et al. (2010), no qual os autores analisaram o sistema de filas de uma panificadora (com uma demanda constante e de proporções consideráveis), no qual há uma única fila para que os clientes paguem suas contas. Os dados foram coletados durante o período de maior movimento do estabelecimento e, a partir desses dados, obteve-se o ritmo de chegada de clientes no sistema por minuto, bem como a frequência de chegada.

Foi constatada a ausência de sazonalidade na demanda e a compatibilidade dos dados com a distribuição de Poisson. A partir da análise de diversos parâmetros (como o número médio de clientes na fila aguardando atendimento e o tempo médio de permanência do cliente no sistema), foi verificada a estabilidade do sistema em questão. Tendo em vista o estudo feito, os autores sugeriram uma melhoria no layout da panificadora a fim de melhorar o nível de satisfação dos clientes e dos funcionários.

Green et al. (2006) utilizaram a Teoria das filas para analisar os atrasos para que as ambulâncias consigam desembarcar pacientes. O objetivo principal do estudo era saber qual a quantidade ideal de funcionários para que se consiga Revista Produção Online, Florianópolis, SC, v. 16, n. 1, p. 210-241, jan./mar. 2016. 
permanecer dentro do padrão máximo de atrasos considerado aceitável (no máximo $20 \%$ dos pacientes poderiam esperar mais de uma hora pelo atendimento). Foi desenvolvido um modelo de regressão logística multivariada e uma análise estatística foi feita.

O estudo demonstrou a possibilidade de melhorar os atendimentos de departamentos de emergência a partir da análise de dados e construção de um modelo de filas que possibilite a redução dos atrasos nos atendimentos e também a redução no número de pacientes que saem sem serem atendidos.

Sheik et al. (2013) desenvolveram um modelo, a partir da Teoria das Filas, no qual demonstram matematicamente a possibilidade de se encontrar as quantidades ideais de filas e de postos de serviços, assim como uma taxa de serviço ótima, através das quais haverá redução no tempo de espera nas filas e, consequentemente, aumento da satisfação dos clientes, devido à maior eficiência do banco.

Romero et al. (2010) fizeram um estudo em uma indústria química, no qual aplicaram a Teoria das Filas para reduzir o tempo de espera para a armazenagem de Paletes, feita com uma empilhadeira. A partir da análise dos dados coletados dos tempos de chegada dos paletes e dos tempos de armazenagens, os autores mostram que a adição de mais uma empilhadeira melhora a eficiência do sistema e aumenta a lucratividade da empresa que era prejudicada com a formação de filas.

\subsection{Características dos Processos de Filas}

Segundo Gross et al. (2008) e Camelo et al. (2010) existem seis características básicas que compõem o processo de filas: processo de chegada de clientes, padrões de serviços prestados, disciplina da fila, capacidade do sistema, número de canais de atendimento e estágios do serviço. As alíneas a seguir realizam uma descrição de cada uma dessas etapas.

\section{a) Processo de chegada de clientes}

Esta característica está relacionada com a distribuição probabilística do tempo entre sucessivas chegadas de clientes. A medição pode ser feita pelo número médio Revista Produção Online, Florianópolis, SC, v. 16, n. 1, p. 210-241, jan./mar. 2016. 
de chegadas por dada unidade de tempo ( $\lambda$ - taxa média de chegada) ou pelo tempo médio entre chegadas sucessivas (IC - intervalo médio de tempo entre chegadas) (GROSS et al., 2008; CAMELO et al., 2010).

\section{b) Padrões de serviço prestados}

O padrão de serviço depende de como o atendimento é realizado, por exemplo, se é feito individualmente ou em grupo. Um mesmo servidor pode atender mais de um cliente ao mesmo tempo (processamento em computadores) ou apenas um (caixa de supermercado). Devido às peculiaridades, o padrão pode ser descrito por duas formas: pela taxa de serviço ( $\mu$ - número de clientes em atendidos em um dado intervalo de tempo) ou pelo tempo de serviço (TA - tempo necessário para atender o cliente) (ibid).

\section{c) Disciplina da Fila}

A disciplina refere-se à forma como os clientes são selecionados para o atendimento quando há existência de fila, ou seja, é o critério estabelecido. Os critérios mais comuns, segundo Gross et al. (2008) e Camelo, et al. (2010) são os seguintes: FIFO (First In, First Out, o primeiro que chega é o primeiro a ser atendido), LIFO (Last In, First Out, o último que chega é o primeiro a ser atendido) PRI (Priority service, atendimento prioritário) e SIRO (Service In Random Order, seleção aleatória de atendimento) (ibid).

\section{d) Capacidade do Sistema}

Trata-se da limitação física do local onde há a prestação de serviço. É o número máximo de usuários dentro do estabelecimento. A capacidade do sistema pode ser considerada finita ou infinita (ibid).

\section{e) Número de canais de atendimento}

Esta característica se refere ao número de estações de serviço em paralelo, Revista Produção Online, Florianópolis, SC, v. 16, n. 1, p. 210-241, jan./mar. 2016. 
que podem atender clientes simultaneamente. O sistema de filas pode dispor de uma ou de múltiplas estações de atendimento, que servem uma ou mais filas (ibid).

\section{f) Estágios do Sistema}

Refere-se ao número de estações de serviço em série, ou seja, trata-se das etapas a serem percorridas pelo cliente até que todo o atendimento seja feito (ibid).

\subsection{Variáveis aleatórias fundamentais}

Um sistema estável de filas, no qual os clientes chegam e aguardam o atendimento dos servidores, apresenta as seguintes variáveis aleatórias fundamentais:

\section{a) Variáveis referentes ao processo de chegada}

$\lambda=$ taxa média de chegada ou ritmo médio de chegada;

IC = intervalo médio entre chegadas.

\section{b) Variáveis referentes à fila}

TF = tempo médio de permanência na fila;

NF = número médio de clientes na fila.

c) Variáveis referentes ao processo de atendimento ou de serviço

TA = tempo médio de atendimento ou de serviço;

$\mathrm{c}=$ capacidade de atendimento ou quantidade de servidores (atendentes);

NA = número médio de transações ou clientes que estão sendo atendidos;

$\mu=$ taxa média ou ritmo médio de atendimento de cada servidor.

\section{d) Variáveis referentes ao sistema (fila + atendimento)}

TS = tempo médio de permanência no sistema;

NS = número médio de transações ou clientes no sistema.

A Tabela 1 apresenta as relações entre as variáveis descritas anteriormente: 
Tabela 1- Relação entre variáveis aleatórias

\begin{tabular}{|c|c|}
\hline Variáveis & Fórmulas \\
\hline Intervalo Entre Chegadas & $I C=1 / \lambda($ Equação 1$)$ \\
\hline Tempo do Atendimento & TA = $1 / \mu$ (Equação 2) \\
\hline $\begin{array}{l}\text { Taxa de Utilização dos } \\
\text { Atendentes }\end{array}$ & $\rho=\lambda / c \mu($ Equação 3) \\
\hline Intensidade de Tráfego & $\mathrm{i}=|\lambda / \mu|=|\mathrm{TA} / \mathrm{IC}|($ Equação 4) \\
\hline \multirow{4}{*}{$\begin{array}{c}\text { Relações entre Fila, Sistema e } \\
\text { Atendimento }\end{array}$} & NS = NF + NA (Equação 5) \\
\hline & $N A=\lambda / \mu($ Equação 6) \\
\hline & $\mathrm{NS}=\mathrm{NF}+\lambda / \mu=\mathrm{NF}+\mathrm{TA} / \mathrm{IC}$ (Equação 7) \\
\hline & TS = TF + TA (Equação 8) \\
\hline \multirow{3}{*}{ Fórmulas de Little } & $N A=\rho=\lambda / c \mu$ (Equação 9) \\
\hline & NF $=\lambda$. TF (Equação 10) \\
\hline & NS $=\lambda$. TS (Equação 11) \\
\hline Ciclo & $\begin{array}{c}\text { Ciclo }=\text { TS }+ \text { TFS (Equação 12) } \\
\text { Ciclo }=\text { Tamanho da População } / \lambda(\text { Equacão 13) }\end{array}$ \\
\hline
\end{tabular}

Fonte: Prado (2014, p. 49)

\subsection{Medidas de desempenho}

Geralmente a medida da efetividade de um sistema de filas é feito por três pontos de vista: 1 - Medida de tempo que um cliente típico é forçado a esperar na fila e no sistema; 2 - Indicação relativa ao acumulo de clientes na fila e no sistema; e 3- Medida de tempo ocioso dos servidores. A Tabela 2 apresenta as variáveis de desempenho de acordo com os 3 pontos de vista supracitados:

Tabela 2- Medidas de desempenho do sistema de filas

\begin{tabular}{l}
\hline Referentes ao sistema \\
\hline TS = Tempo médio que o cliente gasta no sistema \\
Referentes à fila \\
TF = Tempo médio que o cliente gasta na fila de espera \\
NF = Número médio de clientes na fila ou tamanho médio da fila \\
Referente à utilização e ao tempo ocioso dos servidores \\
PO = Índice de ociosidade das instalações \\
$\rho=$ Taxa de utilização dos atendentes
\end{tabular}

Fonte: Prado (2014) 


\subsection{Modelos de distribuição}

Os modelos de distribuição buscam comparar o comportamento das filas no quesito distribuição do tempo de chegadas e do tempo de serviço com distribuições estatísticas conhecidas, para então, prever a chegada de clientes e o tempo de atendimento. Entre os modelos de distribuição com maior citação na literatura estão: Modelo Erlang; Modelo Hiperexponencial; Modelo Determinístico; Modelo Geral e o Modelo exponencial - distribuição de Poisson. Conforme já citado, o presente trabalho pressupõe que as filas analisadas na agência bancária seguem a distribuição de Poisson, detalhado abaixo.

\subsubsection{Modelo exponencial - distribuição de Poisson}

Segundo Prado (2014) este modelo segue a distribuição de Poisson ou exponencial, ou seja, ao se atribuir este modelo ao tempo de chegada está assumindo-se que o processo de chegada de clientes segue o comportamento da distribuição de Poisson. A distribuição de Poisson é uma distribuição de probabilidade discreta que se aplica na ocorrência de eventos em intervalos específicos, sendo que a ocorrência entre os intervalos são independentes - só depende do estado atual. (PRADO, 2014). A Equação 14 apresenta-se a distribuição de Poisson, onde $\lambda$ é a taxa de chegada, $x$ o número, inteiro, de ocorrências e $P(x)$ é a probabilidade em que ocorrem $x$ chegadas por unidade de tempo:

$$
P(x)=\frac{\lambda^{x} e^{-\lambda}}{x !}
$$

\section{PROCEDIMENTOS METODOLÓGICOS}

\subsection{Caracterização da pesquisa}

A caracterização da pesquisa deste trabalho é feita a partir da estrutura apresentada por Ruy (2002) e Martins (1999), com pequenas alterações baseadas nas informações fornecidas por Silva \& Menezes (2001) e Gil (1988), na qual a 
classificação de uma pesquisa pode ser feita segundo quatro critérios: métodos, natureza, objetivos e técnicas utilizadas.

Em relação à classificação Métodos Amplos, a partir da definição de Lakatos \& Marconi (1991), a pesquisa em questão se caracteriza como dedutiva, pois a análise será feita em uma única agência e, a partir das premissas verdadeiras, haverá o estabelecimento de conclusões.

No quesito Procedimentos Técnicos, com base em Gil (1988), este artigo se encaixa como um estudo de caso, pois será feito um estudo aprofundado de uma única agência em consonância com suas peculiaridades, o estudo de caso se justifica pelo enquadramento da análise em "estudos organizacionais e gerenciais", além disso, é feita uma análise de um fenômeno da vida real em que os pesquisadores têm pouco ou nenhum controle (Yin, 2001). E se enquadra como uma pesquisa quantitativa - a partir da definição de Godoy (1995) -, em vista da constante medição de variáveis que descrevem o comportamento das filas bancárias.

Quanto a objetivos, o estudo das filas se caracteriza como descritivo (GIL, 1988). Será avaliado o desempenho e a demanda dos serviços de acordo com a medição de variáveis relacionadas, ou seja, procura-se descrever estes fenômenos com variáveis, característica da pesquisa descritiva.

A pesquisa ainda pode ser classificada como aplicada (SILVA \& MENEZES, 2001), devido ao estudo da demanda e tempo de serviços estarem associados ao desempenho de processos reais, ou seja, problemas específicos. Por fim, a técnica para coleta de dados será por meio de observação direta (RUY, 2002), pois as informações serão coletadas por um funcionário da própria agência bancária.

\subsection{Descrição da agência bancária investigada}

A filial do banco em questão encontra-se dentro de um fórum e possui suas peculiaridades como o atendimento judicial, ausente na maioria das agências bancárias, e o atendimento a um público restrito. Das $10 \mathrm{~h} 00 \mathrm{~m}$ às $12 \mathrm{~h} 30 \mathrm{~m} \mathrm{o}$ atendimento está disponível apenas a advogados e a partir do 12h30m é aberto para o público geral. 
Além da alta demanda judicial, a agência não foge a regra quanto aos negócios e possui o atendimento negocial, destinado ao atendimento de clientes diretos do banco de baixa renda, em paralelo com o atendimento personalizado, destinado a pessoas de renda média, e o atendimento estilo, focado em clientes de alto escalão. Por fim, existe o atendimento caixa, destinado ao atendimento ao público em geral.

A pesquisa visa aplicar a Teoria das Filas, a partir do pressuposto de que as filas analisadas seguem a distribuição de Poisson, com a finalidade de apontar oportunidades de melhorias no tempo de espera dos clientes, melhorando, dessa forma, o nível da prestação de serviços aos clientes. A princípio o estudo na agência envolveria todos os postos de serviços, incluindo o atendimento personalizado e o atendimento estilo. No entanto, após a verificação do baixo nível de demanda e, consequentemente, da baixa formação de filas nos dois atendimentos supracitados, a análise foi simplificada para o atendimento caixa, judicial e negocial. Outra adequação feita foi a divisão das filas de forma a individualizar três filas independentes na análise, pois quem espera para o atendimento judicial, na maioria das vezes, não espera para o atendimento caixa, não havendo sequenciamento ou correlação entre estes serviços.

\subsection{Coleta de dados}

Em relação ao período de apuração, houve a definição de três meses como um escopo interessante para a análise, pois se trata de uma análise estatística, dada a necessidade de um conjunto de dados abrangente para obter um cenário mais próximo da realidade.

A coleta dos dados se deu pelo sistema bancário que, conforme a retirada de senha pelos clientes, registra o horário de chegada, o horário do início do atendimento e o horário do final do atendimento. Esses dados foram coletados diariamente, de cada uma das três filas, em arquivo físico e, posteriormente, foram transcritos em arquivo digital no Microsoft Excel. O início da coleta de dados foi no dia 21 de Novembro de 2014 e o término no dia 13 de Fevereiro de 2015.

Como a agência bancária se encontra dentro de um fórum, seu funcionamento depende do funcionamento do fórum. Por isso, nos dias em que o 
fórum não teve expediente no período analisado, a agência também não teve. Além disso, houve sete dias em que a coleta de dados ficou comprometida devido a problemas técnicos na emissão de senhas. Portanto, diante do período analisado e dos dias com análise comprometida, no total houve a coleta dos tempos de 43 dias. O objeto de estudo são três filas: a fila do atendimento caixa, atendimento judicial e atendimento negocial.

\section{RESULTADOS}

\subsection{Caracterização das filas}

Em posse dos dados e da organização dos mesmos, foi feita a caracterização das três filas seguido do cálculo das variáveis de desempenho, a fim de modelar o funcionamento de cada fila e sua respectiva eficiência. As alíneas a seguir apresentam estes resultados.

\section{a) Processo de chegada de clientes}

A taxa média de chegada no atendimento judicial, negocial e caixa foi calculada nos 43 dias e para alcançar o valor em cada dia foi feito o cálculo de quantos clientes chegaram em cada intervalo de 15 minutos no período de funcionamento da agência (das $10 \mathrm{~h} 00 \mathrm{~m}$ às $16 \mathrm{~h} 00 \mathrm{~m}$ ). Feito o mapeamento dos eventos nos dias da análise, houve o cálculo da taxa média de chegada $(\lambda)$ de cada uma das filas em todos os dias por meio da divisão entre o número total de eventos no dia e a quantidade de intervalos analisados no dia (24 intervalos de 15 minutos). Depois de calculada a taxa média de cada dia, determinou-se a taxa média entre os meses de Novembro à Dezembro, Janeiro à Fevereiro e a taxa média global.

A taxa média global de chegada de clientes no atendimento judicial foi de 2,726 clientes a cada 15 minutos. A taxa média de chegada entre Novembro e Dezembro (3,563 clientes/15 minutos) foi bem superior à taxa entre Janeiro e Fevereiro (1,998 clientes/15 minutos), fato este que demonstra a diferença de demandas nestas épocas e influenciará na análise estatística dos dados. 
Passando para a análise da fila negocial, a taxa média global de chegada de clientes no atendimento judicial foi de 0,900 clientes a cada 15 minutos. As taxas médias de chegada entre Novembro e Dezembro (1,033 clientes/15 minutos) e Janeiro e Fevereiro (0,784 clientes/15 minutos), possuem um valor bem inferior se comparados à fila judicial, ou seja, a demanda de clientes como um todo é inferior, sendo uma média de 3 a 4 clientes por hora. Outra visão interessante é o índice IC, intervalo médio entre chegadas, que medido em todo o período resultou em 16 minutos e 40 segundos, intervalo relativamente alto.

Nos resultados do atendimento caixa destaca-se a regularidade da taxa média de chegada $(\lambda)$. É possível verificar esta característica ao observarem-se as taxas $\lambda$ de Novembro a Dezembro (3,283 clientes/15 minutos), de Janeiro a Fevereiro (3,395 clientes/15 minutos), e a taxa global (3,343 clientes/15 minutos), visto que as três possuem valores muito próximos.

Este comportamento mostra a baixa variabilidade da demanda, no período analisado, ou seja, a necessidade de atendimento é quase constante. Neste caso, a capacidade seria de atender aproximadamente 13 clientes por hora (3,3 $\times 4)$.

Por fim, houve o mapeamento dos dados das três filas e uma abordagem quanto ao processo de chegada de clientes de cada atendimento. Desta abordagem foi possível evidenciar peculiaridades de cada fila.

\section{b) Padrões de serviço prestados}

Primeiramente, o padrão de serviço ou processo de atendimento na área judicial, negocial ou caixa é realizado individualmente, ou seja, um servidor atende uma pessoa por vez. Segundo, esta característica está relacionada à distribuição probabilística dos tempos de atendimentos e pode ser descrita pela taxa de serviço ( $\mu$ ) ou pelo tempo de serviço (TA).

Nesse estudo, houve o cálculo do tempo de atendimento de todos os clientes em 43 dias nas 3 filas. Esta medição foi feita com a diferença entre o horário do final do atendimento e o horário do início de atendimento.

No atendimento judicial, as taxas de serviço apresentam valores próximos nos períodos em 2014 e 2015. Isso comprova que o atendimento judicial é bem padronizado, em outras palavras, o tempo de atendimento não apresenta grandes 
distorções, sendo bem regular. A taxa média de atendimento do período de Novembro a Dezembro foi de 8,025 atendimentos por hora; de Janeiro a Fevereiro foi de 7,040 atendimentos por hora; e a taxa média foi de 7,498 atendimentos por hora.

Em seguida temos as taxas médias de atendimento da fila negocial. Quanto aos resultados do atendimento negocial, verifica-se o baixo índice de atendimentos por hora ( $\mu_{\text {médio }}=4$, aproximadamente). Estes dados demonstram a necessidade de um maior tempo de atendimento (aproximadamente 15 minutos, em média). No atendimento negocial, além de atender as necessidades dos clientes, há um foco em vendas, elementos estes que exigem maior tempo.

Quanto ao atendimento caixa, as taxas de atendimento foram muito maiores se comparadas às filas judicial e negocial. Este dado ilustra a rapidez do atendimento caixa. Nele, os clientes são atendidos de forma rápida (baixo TA), porém há uma demanda grande, sendo, em média, 12 atendimentos por hora.

Desta forma, houve o mapeamento do processo de atendimento das três filas, apresentando resultados e, com estes, notam-se particularidades de cada fila, que podem ser relevantes nas tomadas de decisões gerenciais.

\section{c) Disciplina da Fila}

No atendimento judicial, negocial e caixa a disciplina adotada é a FIFO (First In, First Out), ou seja, o primeiro que entra é o primeiro a ser atendido. Porém, em todas as filas há atendimentos prioritários, como, por exemplo, aos idosos, às pessoas com deficiência, às gestantes entre outros. Esses clientes foram atendidos com antecedência e obtiveram um tempo de espera reduzido. $\mathrm{Na}$ coleta e armazenamento de dados eles foram considerados como os demais, ou seja, foi identificada sua hora de chegada, hora de início e final de atendimento e, posteriormente, foi calculado seu tempo de espera e tempo de atendimento.

\section{d) Capacidade do Sistema}

A capacidade do sistema foi considerada infinita para a análise de todas as 
filas, tendo em vista que nunca foi necessário impedir a entrada de nenhum cliente devido a superlotação.

\section{e) Número de canais de atendimento}

Esta característica se refere ao número de estações que podem atender clientes simultaneamente. Os sistemas judicial, negocial e caixa dispõem de duas estações de atendimento em paralelo servindo uma fila. No caso do atendimento caixa existe uma terceira estação de atendimento reservada apenas para os funcionários do fórum, no entanto, ela foi desprezada nesta pesquisa, pois não há utilização de senhas e, consequentemente, não há computação dos dados.

\section{f) Estágios do Sistema}

Nos três tipos de atendimento analisados, só há uma estação, ou seja, apenas um servidor proporciona o atendimento por completo.

\subsection{Comparação da distribuição do período de chegadas e a distribuição do tempo de serviço do sistema judicial, negocial e caixa com a distribuição probabilística de Poisson}

Nesta pesquisa foi definida a comparação da distribuição do período de chegadas e a distribuição do tempo de serviço do sistema judicial, negocial e caixa com a distribuição probabilística de Poisson (ou distribuição exponencial). Ou seja, busca-se comparar o comportamento das filas e do atendimento com uma distribuição estatística com o intuito de detectar padrões e prever cenários futuros.

\section{a) Distribuição do período de chegadas}

A distribuição de chegadas nas filas judicial, negocial e caixa foi modelada da mesma forma. O processo de chegada foi subdividido em intervalos de 15 minutos e a quantidade de eventos (chegada de clientes) foi calculada em cada intervalo. Este 
mapeamento foi um passo para o cálculo das frequências de chegada, dando início à análise estatística.

Determinado o número de eventos por intervalo, em seguida houve o cálculo da frequência absoluta dos ritmos. O ritmo se trata do número de chegadas em um intervalo de tempo como, por exemplo, a frequência absoluta do ritmo 3 é a quantidade de vezes que ocorreram 3 eventos em um intervalo de 15 minutos.

Após o cálculo da frequência absoluta do ritmo 0 ao 10, determinou-se a frequência relativa por meio da divisão entre a frequência absoluta de cada ritmo pelo total de intervalos (24 intervalos de 15 minutos totalizam 6 horas, expediente de serviço da agência bancária). Este procedimento foi efetuado em 43 dias, nas 3 filas de análise, totalizando 129 distribuições reais de processo de chegada.

A partir da definição das frequências diárias de chegada houve a interpolação destes dados em três grandes escopos de análise: o período entre Novembro e Dezembro, o período entre Janeiro e Fevereiro e o período global entre Novembro de 2014 à Fevereiro de 2015. Para a interpolação das distribuições fez-se a soma das frequências absolutas de cada ritmo, de cada dia de interesse, em determinada fila.

Em seguida, realizou-se o cálculo das frequências relativas por meio da divisão da frequência absoluta pelo número total de intervalos (no caso, foram 1032 intervalos, valor correspondente a multiplicação de 24 intervalos por 43 dias). Com isto, determinou-se a distribuição real de chegada da fila caixa no período global

Em seguida, por meio da aplicação da equação de Poisson (equação 14), sendo $\lambda$ a taxa média de chegada global e $x$ o ritmo de chegadas, houve o cálculo das frequências da distribuição de Poisson.

Com a aplicação deste procedimento nas três filas de análise foi possível comparar seus comportamentos as respectivas distribuições de Poisson associadas. A Figura 1 apresenta a distribuição real e a distribuição de Poisson da fila judicial no período analisado. 
Figura 1 - Distribuição de chegadas global da fila judicial

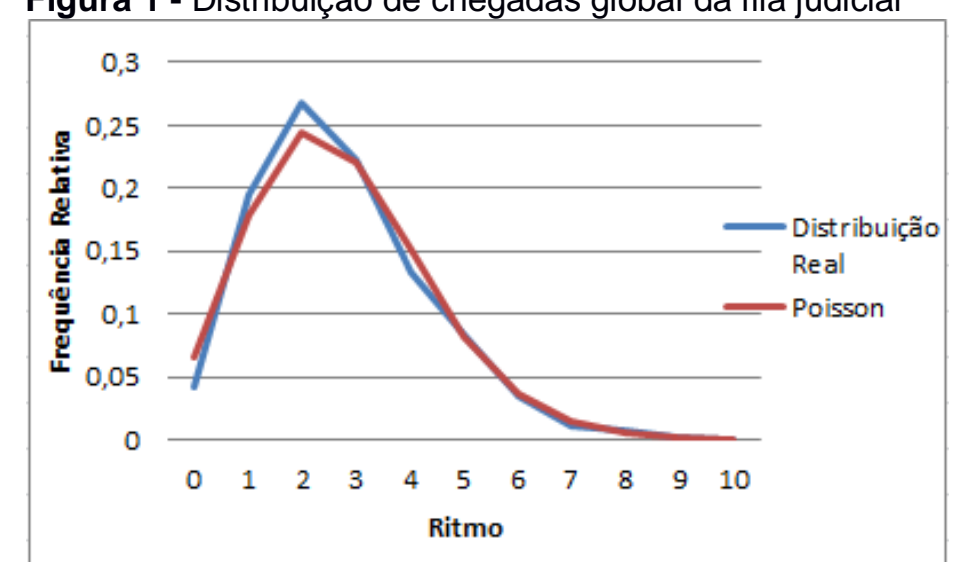

Fonte: Elaborado pelos autores

Por meio da Figura 1 pode-se verificar o comportamento real e de Poisson na chegada de clientes no período global. Nota-se uma aproximação muito grande entre os dados reais e teóricos levando a conclusão que a fila judicial segue a distribuição de Poisson. Esta conclusão é muito relevante, pois permite a gerência da agência se planejar quanto à probabilidade de chegadas utilizando a equação de Poisson.

Por exemplo, na análise da distribuição de Poisson da Figura 1 tem-se que a probabilidade de chegada de até 5 clientes em um intervalo de 15 minutos é de $94,14 \%$ ou a probabilidade de chegada de 6 ou mais clientes é $5,86 \%$. Esses dados são obtidos através da soma das probabilidades de ocorrência de cada ritmo (no caso, do ritmo 0 ao ritmo 5).

Visto os resultados da fila judicial, a seguir, na Figura 2, têm-se os gráficos da distribuição de chegadas na fila negocial: 
Figura 2 - Distribuição de chegadas global da fila negocial

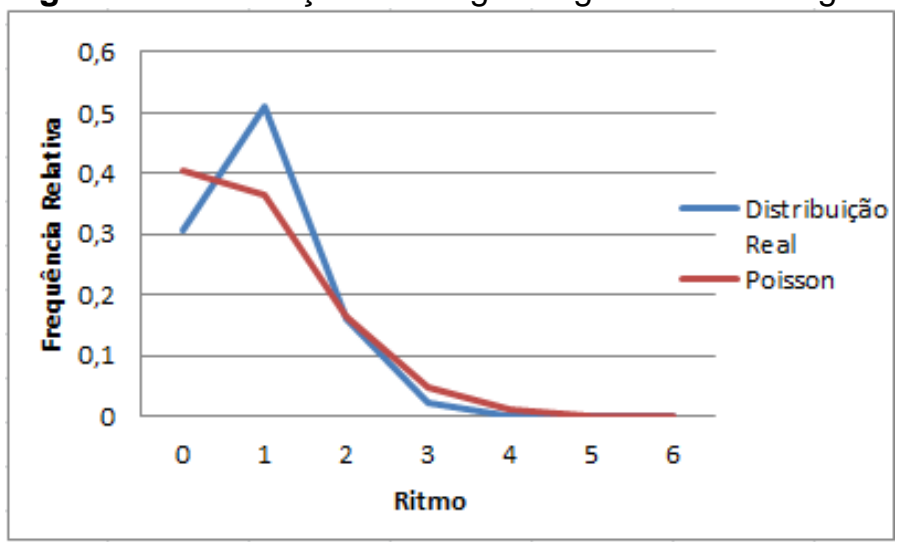

Fonte: Elaborado pelos autores

De forma semelhante à fila judicial, nota-se pela Figura 2 a grande proximidade entre a distribuição real e teórica da fila negocial, chegando à conclusão que esta também segue a distribuição de Poisson. Verifica-se, em comparação à fila judicial, o deslocamento para a esquerda dos gráficos da fila negocial. Isto demonstra uma pequena e desprezível probabilidade de ocorrência de ritmos altos. Por exemplo, na análise teórica global a probabilidade de chegada de até duas pessoas em um intervalo de 15 minutos é de $93,72 \%$ ou a probabilidade de chegada de 3 ou mais pessoas no mesmo intervalo é de 6,28\%. Este dado mostra a baixa probabilidade de ocorrência de filas no atendimento negocial.

Por fim, quanto à fila caixa tem-se o gráfico apresentado na Figura 3.

Figura 3 - Distribuição de chegadas global da fila caixa

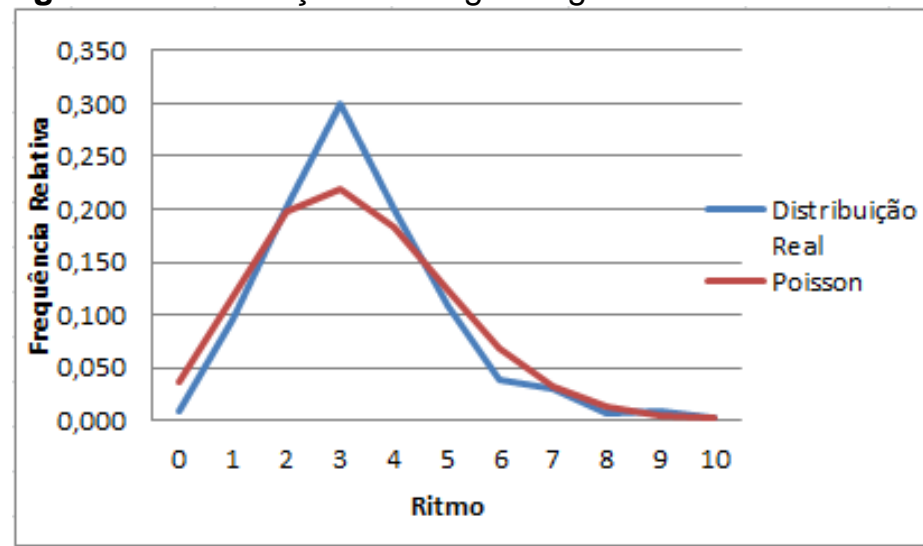

Fonte: Elaborado pelos autores

$\mathrm{Na}$ fila caixa, por meio da análise gráfica, fica evidente que a distribuição real segue o comportamento da distribuição de Poisson, assim como nas filas judicial e 
caixa. Em dados concretos, verifica-se pela distribuição teórica da Figura 3 que a probabilidade de chegada de 1 a 5 pessoas no intervalo de 15 minutos é $84,26 \%$ e a probabilidade de nenhum cliente chegar é 3,5\%, ou seja, a demanda pelo serviço dos caixas é alta e requer atenção. Por fim, conclui-se que a distribuição exponencial, ou de Poisson, ilustra com eficácia as distribuições de chegada das filas judicial, negocial e caixa.

\section{b) Distribuição do tempo de serviço}

A distribuição de atendimento nas filas judicial, negocial e caixa foi modelada nos mesmos padrões. Foram registrados o início e o fim do atendimento em todas as filas. Em posse desses dados, foi calculado o tempo de atendimento para cada cliente. Determinado este tempo para cada atendimento, foi feito o cálculo das frequências absolutas de cada tempo, de 0 a 15 minutos.

Por fim, determina-se a frequência relativa, que corresponde à razão entre a frequência absoluta e o número total de atendimentos. A frequência relativa leva a distribuição estatística do tempo de serviço. Este procedimento foi efetuado em 43 dias, nas 3 filas de análise, resultando em 129 distribuições reais de processo de atendimento.

Com a definição das frequências diárias de atendimento houve a interpolação destes dados em três grandes escopos (semelhante ao processo de chegada): o período entre Novembro e Dezembro, o período entre Janeiro e Fevereiro e o período global entre Novembro de 2014 à Fevereiro de 2015. Para a interpolação das distribuições fez-se a soma das frequências absolutas de cada ritmo, de cada dia de interesse. Após a soma das frequências absolutas, realizou-se o cálculo das frequências relativas, com a finalidade de determinar a distribuição real do atendimento da fila negocial no período global.

Em seguida, por meio da aplicação da equação de Poisson (equação 14) sendo $\mu$ a taxa média de atendimento global e $x$ o ritmo de atendimento - foram calculadas as frequências da distribuição de Poisson.

Com a aplicação deste procedimento nos três serviços foi possível comparar seus comportamentos as respectivas distribuições de Poisson associadas. A seguir 
estão os gráficos com as distribuições reais e de Poisson das filas judicial nos três períodos de análise (Figuras 4, 5 e 6).

Figura 4 - Distribuições globais do atendimento judicial

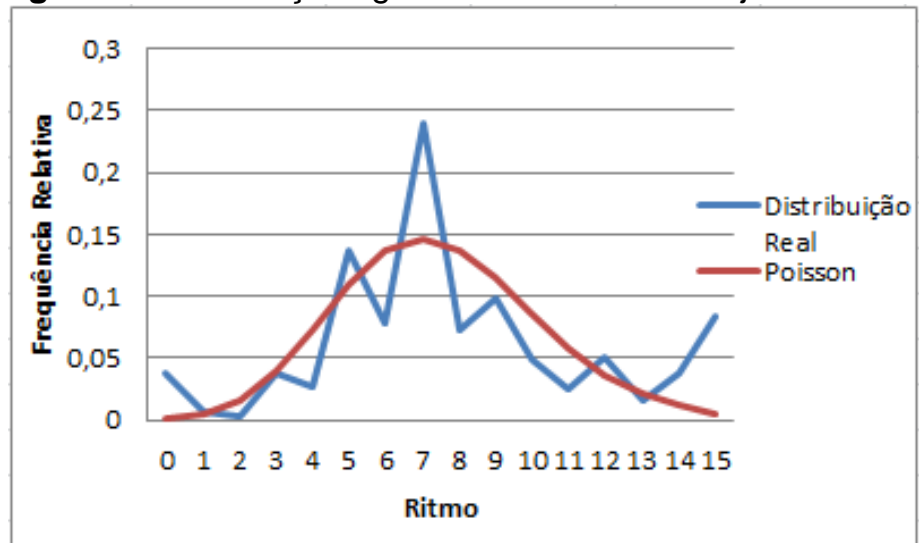

Fonte: Elaborado pelos autores

Verifica-se pela Figura 4 que a distribuição real global segue 0 comportamento da distribuição de Poisson com relativa precisão. Não há a mesma uniformidade que a distribuição teórica, porém a forma do gráfico é a mesma. Por fim, conclui-se que, apesar das distorções, a prestação de serviço judicial pode ser modelada pela distribuição de Poisson, podendo prever o tempo de atendimento neste setor bancário. Quanto ao atendimento negocial, tem-se o gráfico com a distribuição real e teórica deste serviço (Figura 5).

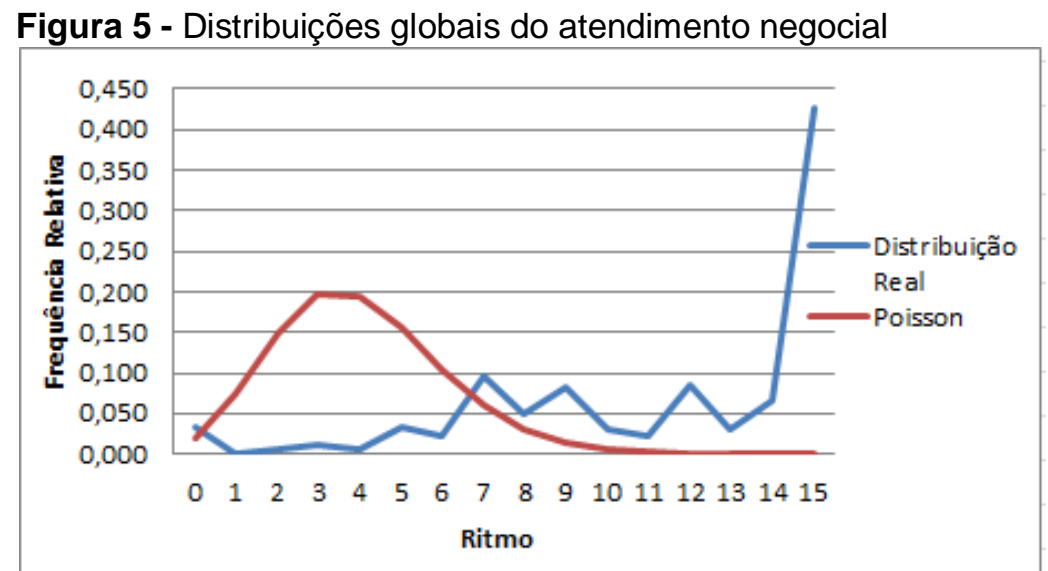

Fonte: Elaborado pelos autores

Através da observação do gráfico da Figura 5, verifica-se que a distribuição exponencial não segue o comportamento da distribuição do atendimento negocial 
sob nenhum aspecto. Portanto, a modelagem deste atendimento deve seguir outros tipos de distribuição ou até mesmo simulação, que fogem ao escopo deste artigo.

Prosseguindo ao atendimento caixa, em seguida, na Figura 6, tem-se o gráfico de sua distribuição real e teórica.

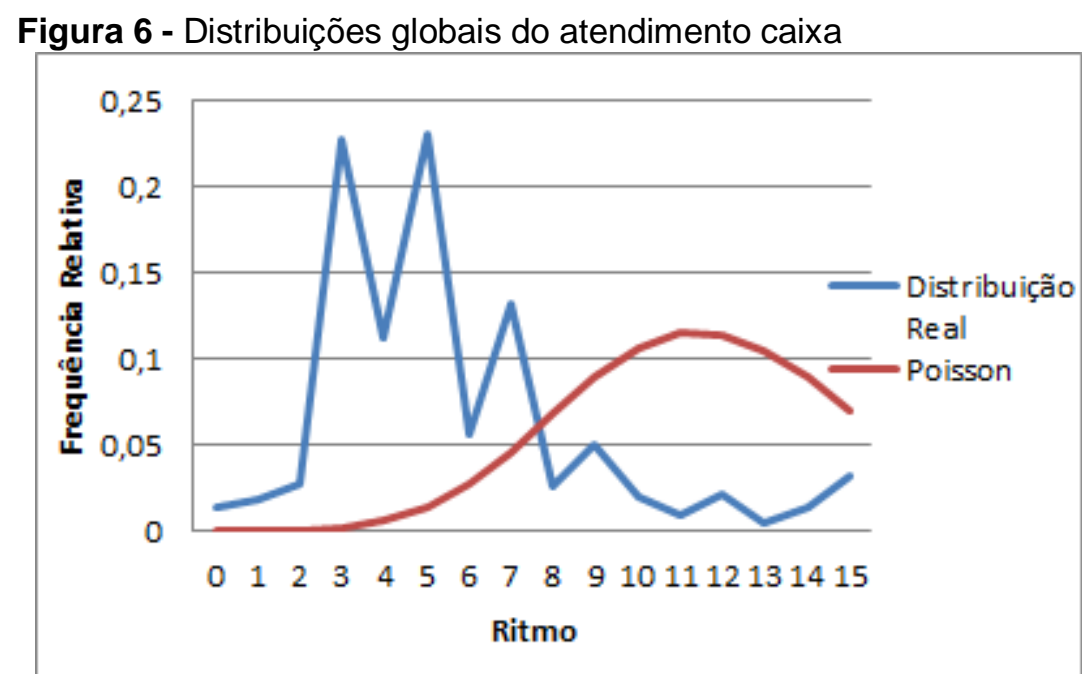

Fonte: Elaborado pelos autores

Por meio da observação do gráfico da Figura 6, nota-se que a distribuição exponencial não segue o comportamento da distribuição do atendimento caixa. Portanto, a modelagem deste atendimento pela distribuição de Poisson também não é possível.

\subsection{Medidas de Desempenho}

Feita a classificação das três filas, outra análise importante da Teoria das Filas é a verificação do desempenho dos sistemas, isto é, a sua eficiência. Esta medida pode apresentar dados concretos e relevantes na orientação dos gestores. A seguir serão apresentados os resultados das medidas de desempenho de cada uma das três filas.

\subsubsection{Atendimento Judicial}

\section{a) Referentes à fila}


Neste segmento são medidos o tempo médio de espera na fila (TF) e o número médio de clientes na fila (NF). A variável TF foi medida todos os dias, em todas as filas. O resultado medido na fila judicial foi de TF (Nov-Dez) $=30$ minutos e 17 segundos, TF (Jan-Dez) = 09 minutos e 25 segundos e TF médio = 19 minutos e 25 segundos. Os resultados demonstram um tempo médio de espera elevadíssimo no período entre Novembro e Dezembro, ou seja, evidencia-se claramente um período crítico no atendimento judicial em que ações devem ser tomadas com urgência.

Quanto ao número médio de clientes na fila (NF) faz-se o cálculo através da Fórmula de Little (Tabela 1, Equação 10). Os resultados obtidos foram:

$$
\begin{aligned}
& \mathrm{NF}_{\text {Nov-Dez }}=7,193 \text { clientes } \\
& \mathrm{NF}_{\text {Jan-Fev }}=1,328 \text { clientes } \\
& \mathrm{NF}_{\text {médio }}=3,529 \text { clientes }
\end{aligned}
$$

O índice NF no fim do ano de 2014 comprova a alta demanda do atendimento judicial neste período, chegando a média de aproximadamente 7 pessoas.

\section{b) Referentes ao sistema}

Esta medida de desempenho engloba à determinação das variáveis TS (tempo médio de permanência no sistema) e NS (número médio de clientes no sistema). A seguir são apresentas as medições de TS, calculados através da soma de TF com TA. Os resultados de TS seguem o mesmo comportamento de TF.

TS $($ Nov-Dez) $=37$ minutos e 49 segundos

$\mathrm{TS}_{(\mathrm{Jan}-\mathrm{Fev})}=18$ minutos e 47 segundos

TS médio $=27$ minutos e 38 segundos

Já o número médio de clientes no sistema (NS) também foi medido conforme a Fórmula de Little (Tabela 1, Equação 10) e foram encontrados os seguintes valores:

$$
\begin{aligned}
& \mathrm{NS}_{\text {Nov-Dez }}=8,983 \text { clientes } \\
& \mathrm{NS}_{\text {Jan-Fev }}=2,502 \text { clientes } \\
& \mathrm{NS}_{\text {médio }}=5,022 \text { clientes }
\end{aligned}
$$

Destaca-se a média de aproximadamente 9 clientes no sistema entre Novembro e Dezembro.

Revista Produção Online, Florianópolis, SC, v. 16, n. 1, p. 210-241, jan./mar. 2016. 


\section{c) Referentes à utilização e ao tempo ocioso dos servidores}

No último segmento verificou-se a taxa de utilização dos atendentes ( $\rho)$ em função dos índices $\lambda, \mu$ e c. A variável c trata da quantidade de servidores, portanto, possui módulo igual a 2 . Apresenta-se a seguir os resultados de $\rho$ no período de análise:

$$
\begin{aligned}
& \rho_{\text {Nov-Dez }}=0,888 \\
& \rho_{\text {Jan-Fev }}=0,568 \\
& \rho_{\text {médio }}=0,727
\end{aligned}
$$

Os valores demonstram que a taxa de utilização dos atendentes no final de 2014 foi muito superior a observada nos dois primeiros meses do ano, a ponto de sobrecarregá-los.

\subsubsection{Atendimento Negocial}

\section{a) Referentes à fila}

O resultado medido na fila negocial, em relação à variável TF, é apresentado a seguir:

$$
\begin{aligned}
& \text { TF } \\
& \text { (Nov-Dez) } \\
& \text { TF } \\
& \text { (Jan-Fev) } 10 \text { minutos e } 41 \text { segundos } \\
& \text { TF } \\
& \text { médio }=10 \text { minutos e } 08 \text { segundos }
\end{aligned}
$$

$\mathrm{Na}$ fila negocial verifica-se que o tempo de espera apresenta uma grande regularidade, tanto que a média entre os meses de 2014 e 2015 ficaram muito próximas. Além disso, o intervalo de valores encontrados se apresentaram satisfatórios ou aceitáveis. Já o número médio de clientes na fila (NF), calculado através da Fórmula de Little (Tabela 1, Equação 10), foram os seguintes:

$$
\begin{aligned}
& \mathrm{NF}_{\text {Nov-Dez }}=0,736 \text { clientes } \\
& \mathrm{NF}_{\text {Jan-Fev }}=0,530 \text { clientes } \\
& \mathrm{NF}_{\text {médio }}=0,623 \text { clientes }
\end{aligned}
$$

Verifica-se que o número médio de clientes na fila negocial sempre é pequeno, sendo abaixo de 1 em todo o período de análise. 


\section{b) Referentes ao sistema}

A seguir são apresentadas as medições de TS (TF + TA):

TS ${ }_{(\text {Nov-Dez) }}=24$ minutos e 48 segundos

$\mathrm{TS}_{(\mathrm{Jan}-\mathrm{Fev})}=26$ minutos e 47 segundos

TS médio $=25$ minutos e 52 segundos

O fato interessante a ser abordado é o aumento, se comparado ao valor de TF, evidenciando ao alto tempo médio de atendimento (TA) do atendimento negocial.

Quanto ao número médio de clientes no sistema (NS) foram encontrados os seguintes valores:

$$
\begin{aligned}
& \mathrm{NS}_{\text {Nov-Dez }}=1,708 \text { clientes } \\
& \mathrm{NS}_{\text {Jan-Fev }}=1,400 \text { clientes } \\
& \mathrm{NS}_{\text {médio }}=1,552 \text { clientes }
\end{aligned}
$$

Destaca-se a baixa média de clientes no sistema negocial, com média de, aproximadamente, 1 a 2 clientes.

\section{c) Referentes à utilização e ao tempo ocioso dos servidores}

A seguir os resultados de $\rho$ no período de 43 dias de análise:

$$
\begin{aligned}
& \rho_{\text {Nov-Dez }}=0,475 \\
& \rho_{\text {Jan-Fev }}=0,427 \\
& \rho_{\text {médio }}=0,451
\end{aligned}
$$

Os valores demonstram que a taxa de utilização dos atendentes não sofreu grandes distorções. Nota-se também o baixo índice se comparada a fila judicial, isto ocorre devido a quantidade de serviços internos, como deferimento de contas e telemarketing.

\subsubsection{Atendimento Caixa}

\section{a) Referentes à fila}


O resultado verificado na fila caixa, em relação a variável TF, é mostrado a seguir:

$$
\begin{aligned}
& \text { TF (Nov-Dez) = } 08 \text { minutos e } 13 \text { segundos } \\
& \text { TF (Jan-Fev) = } 07 \text { minutos e } 52 \text { segundos } \\
& \text { TF médio = } 08 \text { minutos e } 02 \text { segundos }
\end{aligned}
$$

O tempo médio de espera na fila caixa apresentou-se razoável com média de 8 minutos em todo o período. O pico deste índice foi registrado de 16 minutos e 54 segundos.

Já o número médio de clientes na fila (NF), calculado através da Fórmula de Little (Tabela 1, Equação 10), foram os seguintes:

$$
\begin{aligned}
& \mathrm{NF}_{\text {Nov-Dez }}=1,798 \text { clientes } \\
& \mathrm{NF}_{\text {Jan-Fev }}=1,781 \text { clientes } \mathrm{NF}_{\text {médio }}=1,790 \text { cliente }
\end{aligned}
$$

Nota-se que a média de clientes no sistema está sempre entre 1 e 2 clientes, com uma regularidade muito grande durante o período de análise, ou seja, demonstra uma demanda permanente em todo intervalo.

\section{b) Referentes ao sistema}

A seguir apresentam-se as medições do tempo médio no sistema TS (TF + TA):

$$
\begin{aligned}
& \text { TS (Nov-Dez) }=13 \text { minutos e } 47 \text { segundos } \\
& \text { TS }(\text { Jan-Fev) }=12 \text { minutos e } 53 \text { segundos } \\
& \text { TS médio }=13 \text { minutos e } 18 \text { segundos }
\end{aligned}
$$

Os resultados obtidos por TS refletem o comportamento de TF, devido a regularidade do tempo médio na fila (TF) e a regularidade do tempo médio de atendimento (TA). Houve uma média de 13 minutos e 18 segundos durante o intervalo de análise, valor este bem satisfatório para um atendimento de qualidade.

Quanto ao número médio de clientes no sistema (NS) foram encontrados os seguintes valores:

$$
\begin{aligned}
& \mathrm{NS}_{\text {Nov-Dez }}=3,017 \text { clientes } \\
& \mathrm{NS}_{\text {Jan-Fev }}=2,916 \text { clientes } \\
& \mathrm{NS}_{\text {médio }}=2,964 \text { clientes }
\end{aligned}
$$


Novamente verifica-se a regularidade do sistema caixa e também o nível de sua demanda, aproximadamente 3 clientes no sistema. Em paralelo, nota-se que o número médio de clientes nos 3 sistemas analisados seguem a ordem crescente de sistema: negocial, caixa e judicial.

\section{c) Referentes à utilização e ao tempo ocioso dos servidores}

A seguir os resultados de $\rho$ no período de 43 dias de análise:

$$
\begin{aligned}
& \rho_{\text {Nov-Dez }}=0,603 \\
& \rho_{\text {Jan-Fev }}=0,532 \\
& \rho_{\text {médio }}=0,562
\end{aligned}
$$

A taxa de utilização dos atendentes no sistema caixa se apresentou superior se comparada a do atendimento negocial, devido à maior demanda. No entanto, demonstrou-se inferior se comparada a do sistema judicial devido ao atendimento caixa ser mais rápido.

\subsection{Oportunidades de melhorias}

Tomando por base os dados analisados, é possível identificar oportunidades de melhorias a fim de otimizar o tempo de espera nas filas da agência bancária analisada e, dessa forma, melhorar o nível da prestação de serviços aos clientes. Constatou-se que é possível prever o comportamento da chegada dos clientes nas três filas analisadas - dada a semelhança dos dados obtidos com a distribuição de Poisson. O que permite que haja uma organização melhor da agência para receber os clientes.

Além disso, foi observado que enquanto as filas negocial e caixa apresentaram uma demanda bastante regular no período analisado, a fila negocial demonstrou ter uma demanda sazonal. Fato que também facilita o gerenciamento do local.

Em relação ao tempo de serviço, somente a fila judicial apresentou distribuição semelhante à de Poisson, portanto, é a única fila em que é possível prever o tempo de atendimento. Nas demais filas, a modelagem não se mostrou viável.

Revista Produção Online, Florianópolis, SC, v. 16, n. 1, p. 210-241, jan./mar. 2016. 
Com esses dados é possível reorganizar a agência, principalmente nos períodos em que há uma demanda maior para a fila judicial, a qual tem sazonalidade, porém apresenta tempo de atendimento previsível. Como as filas caixa e negocial apresentam demanda regular, apesar de não ser possível modelálas pela distribuição de Poisson, é possível realocar os funcionários dessas filas a fim de não haver sobrecarga na fila judicial.

Adicionalmente, nos períodos em que os funcionários da fila judicial estiverem com ociosidade, é possível redistribuí-los a fim de otimizar os atendimentos das filas negocial e caixa.

\section{CONCLUSÕES}

Com o grau atual de competitividade entre as empresas (GRASEL, 2001; DEIF e ELMARAGHY, 2014) e o nível cada vez maior de exigência dos consumidores (NEGRETTO, 2007), os bancos se vêem com uma necessidade cada vez maior de conquistar e manter clientes. Diante dessa crescente necessidade, a satisfação dos clientes é essencial para que os bancos atinjam seus objetivos.

A satisfação dos clientes é fruto da avaliação que fazem em relação a uma empresa e é diretamente proporcional a lealdade que eles têm em relação à empresa (GUSTAFSSON et al., 2005). As filas enfrentadas pelos consumidores nos bancos reduzem a satisfação dos clientes e, consequentemente, reduzem a lealdade dos mesmos em relação ao banco. Portanto, a redução das filas nas agências é fundamental para aumentar a competitividade das mesmas.

O objetivo do presente artigo foi aplicar a Teoria das Filas a uma agência bancária, a partir do pressuposto de que as filas analisadas seguem a distribuição de Poisson, com a finalidade de apontar oportunidades de melhorias no tempo de espera dos clientes.

O material necessário para a aplicação da Teoria das Filas - horários de chegada, de atendimento e de saída dos clientes das filas judicial, negocial e caixa do banco - foi obtido a partir da coleta de dados do sistema bancário da agência analisada. A partir desse material, aplicou-se a modelagem para analisar os resultados. 
Em relação à distribuição de chegadas dos clientes, verificou-se que a distribuição exponencial, ou de Poisson, ilustra com eficácia essas distribuições das filas: judicial, negocial e caixa. Porém, na análise da distribuição do tempo de serviço, apenas a prestação de serviço judicial pode ser modelada pela distribuição de Poisson, podendo prever o tempo de atendimento bancário. As demais filas analisadas não podem ser modeladas pela distribuição de Poisson.

As medidas de desempenho do atendimento judicial, em relação ao tempo de espera na fila, apresentaram uma grande diferença dentro do período analisado, indicando uma sazonalidade dos atendimentos, com uma demanda maior nos últimos dois meses do ano, quando comparada à demanda dos dois primeiros meses do ano seguinte. O tempo médio de permanência no sistema também foi maior nos meses de novembro e dezembro. Esses dados explicam a sobrecarga dos funcionários, apontada na análise referente à utilização e ao tempo ocioso dos servidores.

$\mathrm{Na}$ fila negocial verifica-se que o tempo de espera apresenta uma grande regularidade, tanto que a média entre os meses de 2014 e 2015 ficaram muito próximas. O tempo de atendimento nesse setor é alto - com uma média maior do que o tempo médio de espera na fila. A média de clientes no sistema negocial foi baixa, aproximadamente, 1 a 2 clientes. A taxa de utilização dos atendentes não apresentou grandes distorções.

O tempo médio de espera na fila caixa apresentou-se razoável com média de 8 minutos em todo o período. Nota-se que a média de clientes no sistema está sempre entre 1 e 2 clientes, com uma regularidade muito grande durante o período de análise. Novamente verificou-se a regularidade do sistema caixa e também o nível de sua demanda, aproximadamente 3 clientes no sistema. A taxa de utilização dos atendentes no sistema caixa se apresentou superior se comparada a do atendimento negocial, devido à maior demanda. No entanto, demonstrou-se inferior se comparada a do sistema judicial devido ao atendimento caixa ser mais rápido.

A partir da análise dos dados, é possível prever o comportamento da chegada dos clientes em todas as filas analisadas. A semelhança das distribuições das chegadas nas três filas com a distribuição de Poisson permite que essa previsão seja feita. Em relação à distribuição do tempo de serviço, somente poderá ser feita a 
modelagem em relação ao atendimento judicial, visto que é o único com distribuição semelhante à de Poisson.

A sazonalidade da demanda no atendimento judicial explica a sobrecarga dos funcionários no período em que há maior procura por esse atendimento. Além disso, os períodos em que houve mais clientes, o tempo médio de permanência no sistema também foi maior. Em contrapartida, tanto a fila negocial como a fila caixa apresentaram comportamentos regulares.

Essas informações são fundamentais, pois permitem à gerência planejar antecipadamente a forma como os atendimentos serão feitos. Evitando tanto o excesso de trabalho para um grupo de funcionários como uma grande ociosidade de parte de seus colaboradores, a partir da redistribuição dos atendentes conforme a análise dos comportamentos das filas.

Mediante ao exposto, conclui-se que a aplicação da Teoria das Filas no setor bancário é viável e pode auxiliar no gerenciamento das agências, melhorando as condições de trabalho e a satisfação dos clientes, com menos filas e um equilíbrio maior. Proporcionando, de fato, vantagens competitivas para a agência.

\section{REFERÊNCIAS}

ARAÚJO, M. A. V. ARAÚJO, F. J. C; ADISSI. P.J. Distribuição da demanda telefônica de um call center através da criação e priorização de filas inteligentes. Revista Produção Online. v. 3, n. 4, 2003. DOI: http://dx.doi.org/10.14488/1676-1901.v3i4.570

BANCO CENTRAL DO BRASIL. Disponível em: <http://www.bcb.gov.br/>. Acesso em 16 jul. 2015.

BANDEIRA, C. R. P. P. ROCHA, S. P. B. Otimização de atendimento bancário: estudo de caso em uma agência bancária em Aracaju-SE. XXX Encontro Nacional de Engenharia de Produção, São Carlos, 2010.

http://www.abepro.org.br/biblioteca/enegep2010 TN STO 114751 15173.pdf

BARBOSA, F. C. O papel dos bancos. Folha de São Paulo, São Paulo, domingo, 05 dez. 2010, Caderno de Mercado. Disponível em: < http://www1.folha.uol.com.br/fsp/mercado/me0512201015.htm >. Acesso em: 15 jul. 2015.

CAMELO, G. R. COELHO, A, S. BORGES, R. M. SOUZA, R. M. Teoria das filas e da simulação aplicada ao embarque de minério de ferro e manganês no terminal marítimo de ponta da madeira. XXX Encontro Nacional De Engenharia De Produção, São Carlos, 2010. http://www.abepro.org.br/biblioteca/enegep2010 th sto 129830 14824.pdf 
CARDOSO F. S. JUNIOR, R. F. F. SANTOS, Y. B. I. Pesquisa operacional: aplicação de Teoria de Filas no sistema de uma panificadora. XXX Encontro Nacional De Engenharia De Produção, São Carlos, 2010.

http://www.abepro.org.br/biblioteca/enegep2010 TN STP 118771 15877.pdf

CHOWDHURY, M. S. R. Queuing theory model used to solve the waiting line of a bank - a study on islami bank bangladesh limited, chawkbazar branch, chittagong. Asian Journal of Social Sciences \& Humanities, v. 2, n. 3, p. 468-478, 2013. http://www.ajssh.leenaluna.co.jp/AJSSHPDFs/Vol.2(3)/AJSSH2013(2.3-51).pdf

DÁVALOS, R. V. Pesquisa Operacional II. Palhoça: Universidade Sul de Santa Catarina, 2012, 26p.

DEIF, A. M.; ELMARAGHY, H. (2014). Cost performance dynamics in lean production leveling. Journal of Manufacturing Systems, v.33, n.4, p. 613-623, 2014. doi:10.1016/j.jmsy.2014.05.010

FERNANDES, S. T. MARINS, F. A. S. APLICAÇÃO DO LEAN SIX SIGMA NA LOGÍSTICA DE TRANSPORTE. Revista Produção Online, v.12, n. 2, p. 297-327, 2012.

DOI: http://dx.doi.org/10.14488/1676-1901.v12i2.763

FERREIRA, M. A. M. JUNIOR, R. M. CARNIERI, C. Análise de desempenho de sistemas portuários usando simulação matemática e estatística. Revista Produção Online, v. 7, n. 3, p. 1-22, 2007. DOI: http://dx.doi.org/10.14488/1676-1901.v7i3.65

FIGUEIREDO, D. D.; ROCHA, S. H. Aplicação da teoria das filas na otimização do número de caixas: um estudo de caso. CESUMAR, v. 12, n. 2, p. 175-182, 2010. http://periodicos.unicesumar.edu.br/index.php/iccesumar/article/view/1300/1113

FREITAS, A. L. P; MORAIS. A. L. C. Análise importância-desempenho aplicada à avaliação da qualidade em serviços bancários. Revista Produção Online. v. 12, n. 4, 2012. DOI: http://dx.doi.org/10.14488/1676-1901.v12i4.1016

FREITAS, C. P. A natureza particular da concorrência bancária e seus efeitos sobre a estabilidade financeira. Economia e Sociedade, Campinas, v.8, p. 51-83, jun. 1997. www.eco.unicamp.br/docprod/downarq.php?id=455\&tp=a

GIL, A. C. Como elaborar projetos de pesquisa. São Paulo: Atlas, 1988, 159 p.

GODOY, A. S. Introdução à pesquisa qualitativa e suas possibilidades. Revista de Administração de Empresas, São Paulo, 1995, n 35, volume 2, pgs 57-63. Disponível em: <http://rae.fgv.br/rae/vol35-num2-1995/introducao-pesquisa-qualitativa-suas-possibilidades>. Acesso em: 14 de outubro de 2015.

GRASEL, D. Padrões, estratégias de competição e competitividade. Revista de estudos sociais, v.3, n.6, p. 59-74, 2001.

http://periodicoscientificos.ufmt.br/index.php/res/article/viewFile/176/166

GREEN, L. V. SOARES, J. GIGLIO, J. F. GREEN, R. A. Using Queueing Theory to Increase the Effectiveness of Emergency Department Provider Staffing. Academic Emergency Medicine, v.13, n.1, 61-68, 2006.

http://sitemaker.umich.edu/emjournalclub/article database/da.data/921326/PDF/singals artic les.pdf

Revista Produção Online, Florianópolis, SC, v. 16, n. 1, p. 210-241, jan./mar. 2016. 
GROSS, D. SHORTLE, J. F. THOMPSON, J. M. HARRIS, C. M. Fundamentals of Queueing Theory. New Jersey: John Wiley \& Sons, 2008.

GUSTAFSSON, A. JOHNSON, M. D. ROOS, I. The Effects of Customer Satisfaction, Relationship Commitment Dimensions, and Triggers on Customer Retention. Journal of Marketing, v.69, p. 210-218, 2005. http://dx.doi.org/10.1509/jmkg.2005.69.4.210

JUNIOR, A. B. BORNIA, A. C. Proposta de um instrumento de medida para avaliar a satisfação de clientes de bancos utilizando a Teoria da Resposta ao Item. Gest. Prod., São Carlos, v.18, n.3, p. 541-554, 2011. http://dx.doi.org/10.1590/S0104-530X2011000300008

LAKATOS, E. M. MARCONI, M. A. Metodologia científica. 2 ed. São Paulo: Atlas, 1991, $247 \mathrm{p}$.

MARTINS, R. A. Sistema de Medição de Desempenho: um modelo para a estruturação do uso. 248 pgs. Tese (Doutorado em Engenharia de Produção). Escola Politécnica da

Universidade de São Paulo. Universidade de São Paulo, São Paulo, 1999.

http://www.dep.ufscar.br/admin/upload/ARTIGO 1150068086.PDF

MULATO, F. M. OLIVEIRA, M. M. B. O impacto de um sistema de agendamento antecipado de docas para carga e descarga na gestão da cadeia de suprimentos. Revista Produção Online, v. 6, n. 3, 2006. DOI: http://dx.doi.org/10.14488/1676-1901.v6i3.631

NARAYAN, B. U. An Introduction to queuing theory: Modeling and Analysis in Applications. Birkhauser, 2008, 268p.

NEGRETTO, R. J. Fidelização de clientes bancários: marketing de relacionamento como base na estratégia de lealdade. 2007. 54 p. Tese (Especialização em Gestão Financeira) - Escola de Administração, Universidade Federal do Rio Grande do Sul, Porto Alegre, 2007. http://hdl.handle.net/10183/14178

OLIVEIRA, M. P. V. Marketing de relacionamento no setor bancário:

A importância do relacionamento de longo prazo para clientes investidores.

2008. 67p. Trabalho de conclusão de curso. Graduação em Ciências

Administrativas, Universidade do Rio Grande do Sul, Porto Alegre, 2008.

http://hdl.handle.net/10183/18024

PAULA, L. F. OREIRO, J. L. BASILIO, F. A. C. Estrutura do setor bancário e o ciclo recente de expansão do crédito: o papel dos bancos públicos federais. Nova Economia, Belo Horizonte, v.23, n.3, p. 473-520, set./dez. 2013. http://dx.doi.org/10.1590/S0103$\underline{63512013000300001}$

PERGHER, I. SILVA, L. A. PACHECA, D. A. J. VACCARO, G. L. R. Análise do impacto da variabilidade de fluxo no dimensionamento de kanbans. Revista Produção Online, v. 14, n. 1, p. 115-142, 2014. DOI: http://dx.doi.org/10.14488/1676-1901.v14.i1.1542

PRADO, D. Teoria das filas e da simulação. Nova Lima: Falconi, 2014, 152 p.

ROMERO, C. M. SALES, D. S. VILAÇA, L. L. CHAVEZ, J. R. A. CORTES, J. M. Aplicação da teoria das filas na maximização do fluxo de paletes em uma indústria química. Revista Eletrônica Pesquisa Operacional para o Desenvolvimento, Rio de Janeiro, v. 2, n.3, p. 226-231, set.-dez. 2010. http://www.podesenvolvimento.org.br/inicio/index.php?journal=podesenvolvimento\&page=art icle\&op=view\&path\%5B\%5D=46\&path\%5B\%5D=97

Revista Produção Online, Florianópolis, SC, v. 16, n. 1, p. 210-241, jan./mar. 2016. 
RUY, M. Aprendizagem organizacional no processo de desenvolvimento de produtos: estudo exploratório em três empresas manufatureiras. 131 p. Dissertação (Mestrado em Engenharia de Produção). Departamento de Engenharia de Produção. Universidade Federal de São Carlos, São Carlos, 2002.

SABBADINI, F. S. GONÇALVES, A. A. OLIVEIRA, M. J. F. A aplicação da teoria das restrições (TOC) e da simulação na gestão da capacidade de atendimento em hospital de emergência. Revista Produção Online, v. 6, n. 3, p. 51-71, 2006.

DOI: http://dx.doi.org/10.14488/1676-1901.v6i3.636

SEBRAE. A competitividade. Disponível em: < http://www.sebraesp.com.br/>. Acesso em: 16 jul. 2015.

SHEIKH, T. SINGH, S. K. KASHYAP, A. K. Application of queuing theory for the improvement of bank service. International Journal of Advanced Computational Engineering and Networking, v.1, n.4, jun. 2013. http://iraj.in/journal/journal file/journal pdf/3-21-139086929015-18.pdf

SILVA, E. L. MENEZES, E. M. Metodologia da Pesquisa e Elaboração da Dissertação. 3 ed. Florianópolis: Laboratório de Ensino a distância da UFSC, 2001, 121 p.

ULLAH, A. IQBAL, K. ZHANG, X-D. AYAT, M. Sub-optimization of Bank Queuing System by Qualitative and Quantitative Analysis. In: Service Systems and Service Management (ICSSSM), 11th International Conference on, Beijing, 2014. DOI: $\underline{10.1109 / I C S S S M .2014 .6874038}$

XIAO, H. ZHANG, G. The Queuing Theory Application in Bank Service Optimization. In: International conference on logistics systems and intelligent management, v.24, p. 1097-1100, 2010. http://dx.doi.org/10.1109/ICLSIM.2010.5461127

YAKUBU, A-W. N. NAJIM, U. An Application of Queuing Theory to ATM Service Optimization: A Case Study. Mathematical Theory and Modeling, v. 4, n. 6, p. 11-23, 2014. http://iiste.org/Journals/index.php/MTM/article/viewFile/13105/13364

YIN, R. Estudo de caso: Planejamento e métodos. 2.ed. Porto Alegre: Bookman, 2001, 290 p.

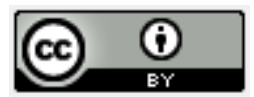

Artigo recebido em 22/07/2015 e aceito para publicação em 31/10/2015

DOI: http://dx.doi.org/ 10.14488/1676-1901.v16i1.2086 